\title{
Influence of a Vibration Isolation System on Planar Dynamics of a Motorcycle
}

\author{
Sudhir Kaul \\ School of Engineering and Technology, Western Carolina University, Cullowhee, NC, 28723.
}

(Received 4 February 2019; accepted 2 August 2019)

This paper examines a model to investigate the impact of a vibration isolation system on the planar (in-plane) dynamics of a motorcycle. While it is not very common, a vibration isolation system is used in some motorcycles to mitigate vibrations resulting from the shaking forces of the engine. For such layouts, the powertrain is assembled to the frame through the vibration isolation system that typically consists of two to four isolators. It is critical to comprehend the influence of the isolation system on the overall dynamic characteristics of the motorcycle due to the coupled dynamics of the rear suspension, the isolation system, and the rear unsprung mass. The influence of a vibration isolation system on the in-plane dynamics is analysed by using a relatively simple model that has been developed in this study. This model has been used to evaluate the influence of the isolation system on natural modes, transmissibility, and ride comfort. Results indicate that the use of a vibration isolation system couples the rear unsprung hop to the pitch motion of the powertrain with a slight increase in the corresponding natural frequency. Results indicate that the use of a vibration isolation system directly affects handling of the motorcycle. Furthermore, results indicate that the pitch of the sprung mass and the hop of the rear unsprung mass are particularly influenced by the vibration isolation system. The model presented in this paper could be useful in the early stages of the design process to compare the rigidly mounted powertrain to different layouts of the vibration isolation system.

\section{NOMENCLATURE}

$\theta$

$\gamma$

$p$

$b$

$k_{1 f y}, k_{1 r y}$,

$k_{1 f x}, k_{1 r x}$

$c_{1 f y}, c_{1 r y}$,

$c_{1 f x}, c_{1 r x}$

$F_{x r}, F_{y r}$

$x_{r 1}, y_{r 1}$

$x_{f 1}, y_{f 1}$

$y, y_{f}, y_{r}$,

$y_{p}$

$c_{f}, c_{r}$

$k_{f}, k_{r}$

$m, I$

$m_{p}, I_{p}$
Sprung mass pitch

Powertrain pitch

Wheelbase

Distance from centre of mass of spring mass to rear tire

Stiffness constants of front and rear vibration isolators

Damping constants of front and rear vibration isolators

Interaction force between rear unsprung mass and swing arm

Position of front and rear vibration isolators

Vertical displacement of the sprung mass, front unsprung mass, rear unsprung mass, and powertrain respectively

Equivalent damping constants

Equivalent stiffness constants

Mass and mass moment of inertia of sprung mass

Mass and mass moment of inertia of powertrain

\section{INTRODUCTION}

The study of motorcycle dynamics has been an active area of research for the past few decades. ${ }^{1}$ Research has ranged from the investigation of stability and control to the enhancement of the suspension system. ${ }^{2,3}$ The dynamic characteristics in multiple states of motion have been investigated in the literature to comprehend performance, handling, stability, etc. ${ }^{4}$ Similarities between some of the key characteristics of bicycle and motorcycle dynamics have also been investigated by researchers to understand phenomenon such as weave, wobble, steering, instability, etc. ${ }^{5}$ Commercial programs such as BikeSim and
Fastbike have enabled researchers to analyse configurations of dynamic models to investigate such aspects of motorcycle dynamics as stability, handling, manoeuvrability, and in-plane dynamics. ${ }^{6,7}$ Comprehensive models have been developed to understand characteristics of multi-body dynamics of a motorcycle, and results have been found to be comparable to test data and rider experiences. ${ }^{2,5}$ While the multi-body dynamics models are extremely useful for design and analysis at the system level, their use is somewhat limited for component level design of parts such as vibration isolators. Some of the research in motorcycle dynamics has focused on modelling special purpose motorcycles that are used for off-road or racing purposes, these systems require modelling adjustments since road irregularities induce extreme excursions that may result in nonlinear response. $^{8}$

Most of the existing models can be broadly divided into two categories: in-plane and out-of-plane. The out-of-plane models primarily attempt to comprehend handling, manoeuvrability, stability, and control aspects associated with the motion of the motorcycle. The in-plane models typically investigate ride comfort at constant speeds under straight-running conditions along with the influence of road profiles and suspension characteristics. The broad division of the models into the two categories allows a designer to focus on certain components and attributes that are associated with specific aspects of motorcycle dynamics. Although the in-plane modes are generally decoupled from the out-of-plane modes, aspects of in-plane dynamics directly affect the out-of-plane dynamics and vice versa.

Although the use of vibration isolation systems is not widespread in motorcycles, there are some commercial manufacturers who use isolators to mitigate shaking forces that are transmitted to the frame. ${ }^{9,10}$ Most manufacturers use a rigidly mounted powertrain without the use of an isolation system, this is primarily accomplished by engine balancing that results in significantly reducing shaking forces. The use of a vibration 
isolation system significantly affects the overall stiffness of the chassis and in turn affects the handling of the motorcycle, requiring design changes to enhance stiffness through alternative means. ${ }^{9,11}$

This study attempts to comprehend the role played by the vibration isolation system in changing the in-plane dynamics of a motorcycle. The model developed in this study holistically assesses ride comfort of the system by reviewing the role of the isolation system in conjunction with the suspension system. The proposed model is particularly useful for comparing the in-plane dynamics of a motorcycle with an isolation system to a rigidly mounted powertrain, thereby allowing a designer to comprehend the influence of the isolation system and make design changes, if needed, to key design parameters.

In this paper, Section 2 presents the proposed model and a discussion on the main assumptions associated with this model. The proposed model has been used for multiple simulations that are presented in Section 3. Overall conclusions from the analysis of the simulation results are drawn in Section 4.

\section{MODEL-PLANAR DYNAMICS}

The governing equations of motion (EOM) for the model are presented in this section along with a description of the model and the relevant assumptions associated with the model. The model is divided into four rigid bodies that are interconnected either through the suspension system or the vibration isolation system. Each suspension system and each vibration isolator are represented as a spring damper unit. A layout of the model is shown in Fig. 1.

The rigid body representing the chassis and the rider combined together has been modelled with two degrees-offreedom (DOF), this rigid body is called as the sprung mass. It may be noted that no distinction has been made between the chassis and the rider, the rider is assumed to be rigidly attached. The EOM for the sprung mass are derived to be as follows:

$$
\begin{aligned}
m \ddot{y}+ & \left(k_{f}+k_{r}+k_{1 f y}+k_{1 r y}\right) y-k_{f} y_{f}-k_{r} y_{r}+ \\
& {\left[k_{f}(p-b)-k_{r} b+k_{1 f y} x_{f 1}-k_{1 r y} x_{r 1}\right] \theta+} \\
& \left(k_{1 r y} x_{r 1}-k_{1 f y} x_{f 1}\right) \gamma+\left(-k_{1 f y}-k_{1 r y}\right) y_{p}+ \\
& \left(c_{f}+c_{r}+c_{1 f y}+c_{1 r y}\right) \dot{y}-c_{f} \dot{y}_{f}-c_{r} \dot{y}_{r}+ \\
& {\left[c_{f}(p-b)-c_{r} b+c_{1 f y} x_{f 1}-c_{1 r y} x_{r 1}\right] \dot{\theta}+} \\
& \left(c_{1 r y} x_{r 1}-c_{1 f y} x_{f 1}\right) \dot{\gamma}+\left(-c_{1 f y}-c_{1 r y}\right) \dot{y}_{p}=0 ; \\
I \ddot{\theta}+ & {\left[k_{f}(p-b)^{2}+k_{r} b^{2}+k_{1 f y} x_{f 1}^{2}+k_{1 r y} x_{r 1}^{2}\right] \theta+} \\
& {\left[k_{f}(p-b)-k_{r} b+k_{1 f y} x_{f 1}-k_{1 r y} x_{r 1}\right] y-} \\
& k_{f}(p-b) y_{f}+k_{r} b y_{r}+\left(k_{1 r y} x_{r 1}-k_{1 f y} x_{f 1}\right) y_{p}+ \\
& \left(-k_{1 f y} x_{f 1}^{2}-k_{1 r y} x_{r 1}^{2}\right) \gamma+ \\
& {\left[c_{f}(p-b)^{2}+c_{r} b^{2}+c_{1 f y} x_{f 1}^{2}+c_{1 r y} x_{r 1}^{2}\right] \dot{\theta}+} \\
& {\left[c_{f}(p-b)-c_{r} b+c_{1 f y} x_{f 1}-c_{1 r y} x_{r 1}\right] \dot{y}-} \\
& c_{f}(p-b) \dot{y}_{f}+c_{r} b \dot{y}_{r}+\left(c_{1 r y} x_{r 1}-c_{1 f y} x_{f 1}\right) \dot{y}_{p}+ \\
& \left(-c_{1 f y} x_{f 1}^{2}-c_{1 r y} x_{r 1}^{2}\right) \dot{\gamma}=0 .
\end{aligned}
$$

In Eq. (1), $m$ is the sprung mass that consists of the chassis and the rider and in Eq. (2), $I$ is the mass moment of inertia of the sprung mass about the $z$-axis passing through the centre of mass, as per the coordinate system in Fig. 1. The vertical displacement (bounce) of the sprung mass is represented by $y$ and the in-plane (pitch) rotation of the sprung mass is represented by $\theta$. Furthermore, $p$ is the wheelbase (distance between the contact point of the front tire and the contact point of the rear tire) and $b$ is the distance from the centre of mass of the sprung mass to the contact point of the rear tire. It may be noted that $\left(x_{r 1}, y_{r 1}\right)$ identifies the position of the rear isolator while $\left(x_{f 1}, y_{f 1}\right)$ identifies the position of the front isolator. In Eq. (1) and (2), $k_{f}$ and $c_{f}$ are the equivalent stiffness and damping constants of the front suspension, and $k_{r}$ and $c_{r}$ are the equivalent stiffness and damping constants of the rear suspension. The equivalent stiffness and damping constants have been calculated from suspension characteristics as well as the suspension layout; these steps can be directly referenced from the literature. ${ }^{12}$ Also, $y_{f}$ and $y_{r}$ are the vertical displacements of the front and rear unsprung mass respectively, also called as the hop motion of the unsprung masses. It may be noted that each unsprung mass is the combined mass of the wheel and other parts connected to the wheel such as the tire, brake, axle, and related hardware. Also, $k_{1 r y}$ and $c_{1 r y}$ are the vertical stiffness and damping constants of the rear isolator and $k_{1 f y}$ and $c_{1 f y}$ are the stiffness and damping constants of the front isolator in the vertical direction, as seen in Fig. 1.

The front unsprung mass $\left(m_{f}\right)$ has been modelled with only one degree-of-freedom while the rear unsprung mass $\left(m_{r}\right)$ has been modelled with two DOF. This is because the rear unsprung mass is attached to the powertrain through the swing arm, connected through a pin joint on the powertrain and a pin joint at the rear axle, as shown in the schematic of the model in Fig. 1. The front unsprung mass is only connected to the front suspension. The EOM for the front unsprung mass and the rear unsprung mass are derived as:

$$
\begin{aligned}
& m_{f} \ddot{y}_{f}-k_{f} y-k_{f}(p-b) \theta+\left(k_{f}+k_{p f}\right) y_{f}-c_{f} \dot{y}- \\
& \quad c_{f}(p-b) \dot{\theta}+\left(c_{f}+c_{p f}\right) \dot{y}_{f}=0 ; \\
& m_{r} \ddot{x}_{r}=F_{x r} ; \\
& m_{r} \ddot{y}_{r}+\left(k_{p r}+k_{r}\right) y_{r}-k_{r} y+k_{r} b \theta+\left(c_{p r}+c_{r}\right) \dot{y}_{r}- \\
& \quad c_{r} \dot{y}+c_{r} b \dot{\theta}=F_{y r} .
\end{aligned}
$$

In Eqs. (3), (4) and (5), $y_{r}$ and $x_{r}$ are the vertical and foreaft displacements of the rear unsprung mass while $y_{f}$ is the vertical displacement of the front unsprung mass. Also, $k_{p f}$ and $c_{p f}$ are the equivalent stiffness and damping constants of the front tire, and $\mathrm{kpr}$ and $c_{p r}$ are the equivalent stiffness and damping constants of the rear tire. Furthermore, $F_{x r}$ and $F_{y r}$ are the interaction forces between the rear unsprung mass and the swing arm, resulting from the swing arm connection at the rear axle.

The powertrain has been modelled as a rigid body with three DOF-two translational and one rotational (pitch) degree-offreedom, represented by $x_{p}, y_{p}$ and $\gamma$ respectively. ${ }^{13}$ Since the stiffness of the isolators is significantly lower than the stiffness of the frame, the powertrain mass and inertia have been separated from the sprung mass. It is common to enhance the chassis stiffness of isolated systems by alternate means since the powertrain stiffness does not directly contribute to the overall stiffness of the frame as a rigidly attached part of the sprung mass. $^{14}$

While the front end of the powertrain is connected to the sprung mass through a vibration isolator, it is possible to model this alternately. ${ }^{9,11}$ The rear end of the powertrain is connected to the sprung mass through another isolator while being connected to the rear unsprung mass through a pivot connection. The swing arm (commonly called the rear fork) connects the rear wheel to the frame and powertrain, and is attached through a revolute joint. ${ }^{11}$ It may be noted that the powertrain could be assembled to the frame through more than two isolators, the 


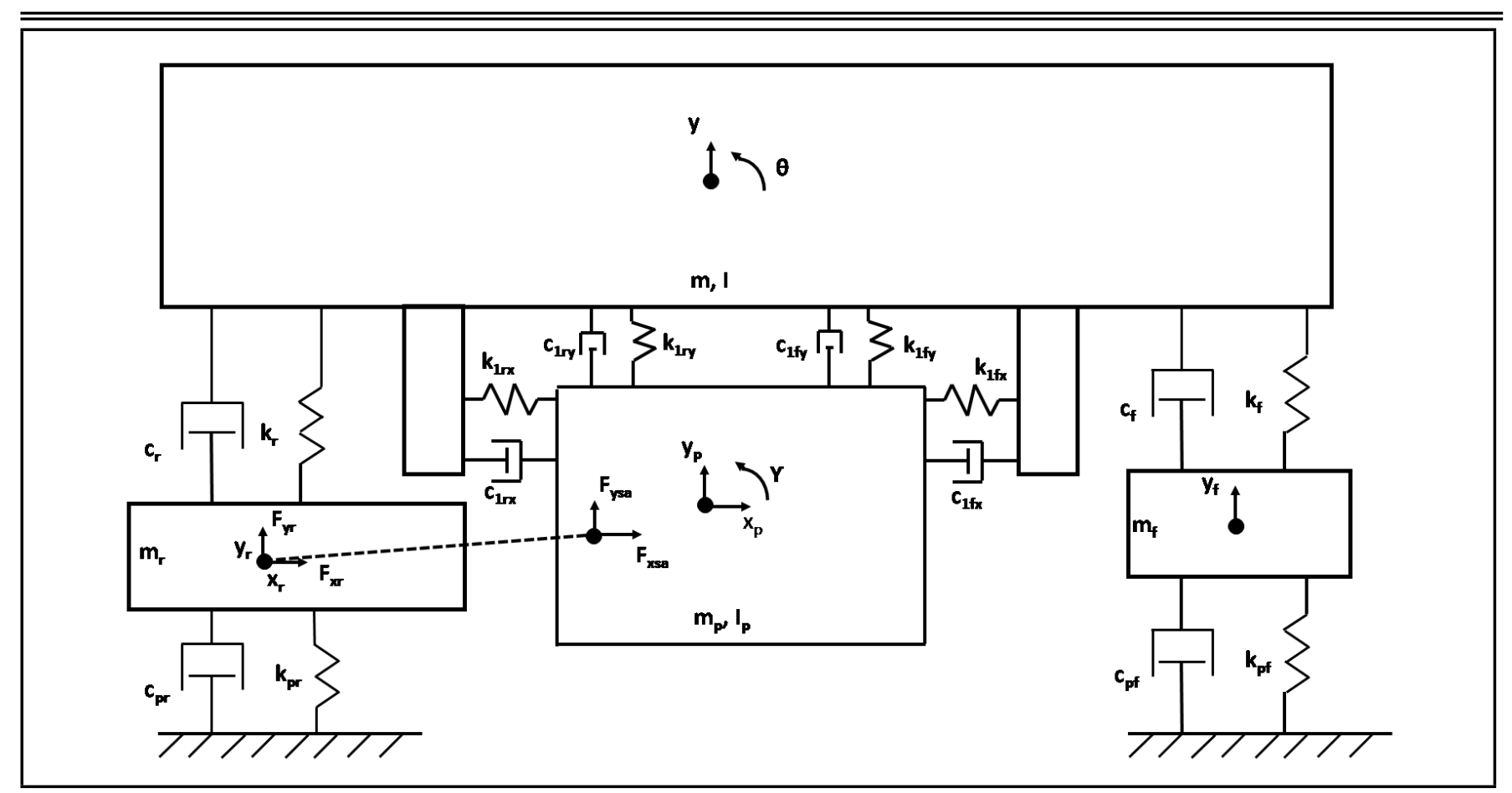

Figure 1. Eight degree-of-freedom model.

EOM can be suitably modified to accommodate more isolators, if needed, while still retaining the overall model discussed in this section. The EOM for the powertrain are derived to be as follows:

$$
\begin{aligned}
& m_{p} \ddot{x}_{p}+\left(k_{1 f x}+k_{1 r x}\right) x_{p}+\left(k_{1 r x} y_{r 1}-k_{1 f x} y_{f 1}\right) \gamma+ \\
& \quad\left(c_{1 f x}+c_{1 r x}\right) \dot{x}_{p}+\left(c_{1 r x} y_{r 1}-c_{1 f x} y_{f 1}\right) \dot{\gamma}=F_{x s a} ; \\
& m_{p} \ddot{y}_{p}+\left(k_{1 f y}+k_{1 r y}\right) y_{p}+\left(k_{1 f y} x_{f 1}-k_{1 r y} x_{r 1}\right) \gamma+ \\
& \quad\left(-k_{1 f y} x_{f 1}+k_{1 r y} x_{r 1}\right) \theta+\left(-k_{1 f y}-k_{1 r y}\right) y+ \\
& \quad\left(c_{1 f y}+c_{1 r y}\right) \dot{y}_{p}+\left(c_{1 f y} x_{f 1}-c_{1 r y} x_{r 1}\right) \dot{\gamma}+ \\
& \quad\left(-c_{1 f y} x_{f 1}+c_{1 r y} x_{r 1}\right) \dot{\theta}+\left(-c_{1 f y}-c_{1 r y}\right) \dot{y}=F_{y s a} ; \\
& I_{p} \ddot{\gamma}+\left(k_{1 f x} y_{f 1}^{2}+k_{1 f y} x_{f 1}^{2}+k_{1 r x} y_{r 1}^{2}+k_{1 r y} x_{r 1}^{2}\right) \gamma+ \\
& \quad\left(k_{1 r x} y_{r 1}-k_{1 f x} y_{f 1}\right) x_{p}+\left(k_{1 f y} x_{f 1}-k_{1 r y} x_{r 1}\right) y_{p}+ \\
& \quad\left(-k_{1 f y} x_{f 1}+k_{1 r y} x_{r 1}\right) y+\left(-k_{1 f y} x_{f 1}^{2}-k_{1 r y} x_{r 1}^{2}\right) \theta+ \\
& \quad\left(c_{1 f x} y_{f 1}^{2}+c_{1 f y} x_{f 1}^{2}+c_{1 r x} y_{r 1}^{2}+c_{1 r y} x_{r 1}^{2}\right) \dot{\gamma}+ \\
& \quad\left(c_{1 r x} y_{r 1}-c_{1 f x} y_{f 1}\right) \dot{x}_{p}+\left(c_{1 f y} x_{f 1}-c_{1 r y} x_{r 1}\right) \dot{y}_{p}+ \\
& \quad\left(-c_{1 f y} x_{f 1}+c_{1 r y} x_{r 1}\right) \dot{y}+\left(-c_{1 f y} x_{f 1}^{2}-c_{1 r y} x_{r 1}^{2}\right) \dot{\theta}= \\
& \quad F_{x s a} y_{s a}-F_{y s a} x_{s a} .
\end{aligned}
$$

In Eqs. (6), (7) and (8), the front and rear isolator locations are represented as $\left(x_{f 1}, y_{f 1}\right)$ and $\left(x_{r 1}, y_{r 1}\right)$ respectively, with respect to the centre of mass of the powertrain. Also, $m_{p}$ is the mass of the powertrain and $I_{p}$ is the mass moment of inertia of the powertrain along the $z$-axis about its centre of mass. Furthermore, $F_{x s a}$ and $F_{y s a}$ are the interaction forces at the powertrain due to the swing arm connection between the powertrain and the rear axle while $\left(x_{s a}, y_{s a}\right)$ is the location of the swing arm connection point from the centre of mass of the powertrain. In Eqs. (6), (7) and (8), $k_{1 r x}$ and $c_{1 r x}$ are the stiffness and damping constants of the rear isolator in the foreaft direction and $k_{1 f x}$ and $c_{1 f x}$ are the stiffness and damping constants of the front isolator in the fore-aft direction.

The EOM from Eqs. (1) through (8) are compiled to identify the mass $(M)$, damping $(C)$, and stiffness $(K)$ matrices of the system, all three matrices are $8 \times 8$. Using base excitation due to road irregularity at the front and rear tires, displacement transmissibility is computed from the EOM as the ratio between the output and the excitation input. For the model developed in this study, displacement transmissibility due to external excitation is derived as:

$$
T=\left[-\omega^{2} M+i \omega C+K\right]^{-1}[\dot{K}+i \omega \dot{C}] .
$$

In Eq. (9), $T$ is the $8 \times 1$ transmissibility matrix, $\omega$ is the excitation frequency, while $K^{\prime}$ and $C^{\prime}$ are $8 \times 1$ matrices resulting from the source of base excitation.

In order to solve for the system response in time domain, the EOM from Eqs. (1) to (8) are compiled in a state space model that is expressed as follows:

$$
\left[\begin{array}{c}
\dot{X}_{1} \\
\dot{X}_{2}
\end{array}\right]=\left[\begin{array}{cc}
Z_{8,8} & I_{8,8} \\
-M^{-1} K & -M^{-1} C
\end{array}\right]\left[\begin{array}{c}
X_{1} \\
X_{2}
\end{array}\right]+\left[\begin{array}{c}
Z_{8,1} \\
M^{-1} F
\end{array}\right]
$$

In Eq. (10), $X_{1}=\left[\begin{array}{llllllll}y & \theta & y_{f} & x_{r} & y_{r} & x_{p} & y_{p} & \gamma\end{array}\right]^{T}$ is the $8 \times 1$ matrix and $\dot{X}_{1}=X_{2} . Z_{8,8}$ and $Z_{8,1}$ are $8 \times 8$ and $8 \times 1$ zero matrices, respectively, while $I_{8,8}$ is an $8 \times 8$ identity matrix. Also, $F$ is the time varying force matrix that consists of input force that serves as external excitation to the system that is transmitted through the front and rear tires.

In order to evaluate ride comfort, the frequency response function (FRF) for accelerations of all DOF resulting from excitation at the front and rear tires is computed from the EOM listed in Eqs. (1) through (8), the FRF matrix for the system, $H(\omega)$, is expressed as:

$$
H(\omega)=-\omega^{2}\left[-\omega^{2} M+i \omega C+K\right]^{-1}[\hat{K}+i \omega \hat{C}] .
$$

In Eq. (11), $\hat{K}$ and $\hat{C}$ are $8 \times 2$ matrices. $H(\omega)$ is also $8 \times 2$ and computes the accelerations of all DOF due to excitation at the front tire as well as the rear tire in the two columns of the matrix. Since there is an inherent delay between the inputs at the front tire and the rear tire due to irregularities of the road surface, the frequency response is computed as: ${ }^{3}$

$$
\ddot{X}_{1}(\omega)=H(\omega)\left[\begin{array}{c}
1 \\
e^{-i \omega \frac{p}{V}}
\end{array}\right] Y_{f i}(\omega)=H^{*}(\omega, V) Y_{f i}(\omega) .
$$


In Eq. (12), $Y_{f i}(\omega)$ is the input at the front tire in frequency domain, $\mathrm{p}$ is the wheelbase as defined in the EOM, and $V$ is the constant speed at which the motorcycle is traveling in a straight line. The exponential term in Eq. (12) arises from the time delay between the input at the two tires and is a function of the wheelbase as well as the motorcycle speed, the phenomenon is commonly referred to as wheelbase filtering, ${ }^{3} H^{*}(\omega, V)$ is the modified FRF that accounts for wheelbase filtering.

In order to account for a random road profile, power spectral density (PSD) has been used to evaluate ride comfort and handling from acceleration levels. The capability of PSD to represent the random profile of a road surface is well documented in the literature. ${ }^{15}$ The acceleration PSD for each DOF can be computed as follows:

$$
S_{i i}(\omega, V)=\left|H_{i}^{*}(\omega, V)\right|^{2} S_{r r}(\omega, V) .
$$

In Eq. (13), $S_{r r}(\omega, V)$ is the PSD of the road profile and $S_{i i}(\omega, V)$ is the PSD of acceleration of each DOF (where, $\left.i=y \quad \theta \quad y_{f} x_{r} y_{r} x_{p} y_{p} \gamma\right)$ that is computed by using the magnitude of the modified FRF, $H^{*}(\omega, V)$, corresponding to each DOF. The PSD of the road profile has been computed by assuming a constant speed of the motorcycle on a good road surface. ${ }^{3,15}$

The model presented in this section has a total of eight degrees-of-freedom and has been used for multiple simulations in Section 3. Simulation results have been discussed in Section 3 and the parameters for the model have been identified from relevant literature.

\section{RESULTS}

The simulation results for the model presented in Section 2 are presented in this section. Most of the motorcycle parameters have been taken from existing literature ${ }^{12}$ and the isolator parameters have been taken from another study. ${ }^{13}$ The following values have been used for all the simulations presented in this section: $p=1.4 \mathrm{~m}, b=0.7 \mathrm{~m}, m=200 \mathrm{~kg}, I=$ $38 \mathrm{kgm}^{2}, k_{f}=15 \mathrm{kN} / \mathrm{m}, k_{r}=24 \mathrm{kN} / \mathrm{m}, k_{p f}=180 \mathrm{kN} / \mathrm{m}$, $k_{p r}=180 \mathrm{kN} / \mathrm{m}, m_{f}=15 \mathrm{~kg}, m_{r}=18 \mathrm{~kg}, m_{p}=125 \mathrm{~kg}$, $I_{p}=8 \mathrm{kgm}^{2}, x_{r 1}=320 \mathrm{~mm}, y_{r 1}=55 \mathrm{~mm}, x_{f 1}=325 \mathrm{~mm}$, $y_{f 1}=20 \mathrm{~mm}, k_{1 r y}=357.6 \mathrm{~N} / \mathrm{mm}, k_{1 r x}=410.9 \mathrm{~N} / \mathrm{mm}$, $k_{1 f y}=258.7 \mathrm{~N} / \mathrm{mm}, k_{1 f x}=358.5 \mathrm{~N} / \mathrm{mm}, x_{s a}=270 \mathrm{~mm}$, $y_{s a}=30 \mathrm{~mm}$. The model developed in Section 2 is used to calculate natural frequencies, mode shapes and the corresponding damping ratios. The model is also used to determine the system response to inputs transmitted from the road surface through the front and rear tires.

\subsection{Natural modes}

The first simulation is performed for an undamped system with the powertrain being rigidly attached to the sprung mass, this reduces the model to a five degree-of-freedom system since the powertrain becomes a part of the sprung mass. The undamped natural frequencies for this model are: $1.65 \mathrm{~Hz}$ for the vertical (bounce) mode of the sprung mass along with the powertrain mass, $16.85 \mathrm{~Hz}$ for the vertical (hop) mode of the rear unsprung mass, $18.15 \mathrm{~Hz}$ for the vertical (hop) mode of the front unsprung mass, $580.85 \mathrm{~Hz}$ for the fore-aft mode of the rear unsprung mass, and $915.71 \mathrm{~Hz}$ for the sprung mass pitch. This simulation is used as a baseline and is followed by the analysis of an undamped system when the powertrain is coupled to the sprung mass through the vibration isolation system, as modelled in Section 2. The first three frequencies from rigid

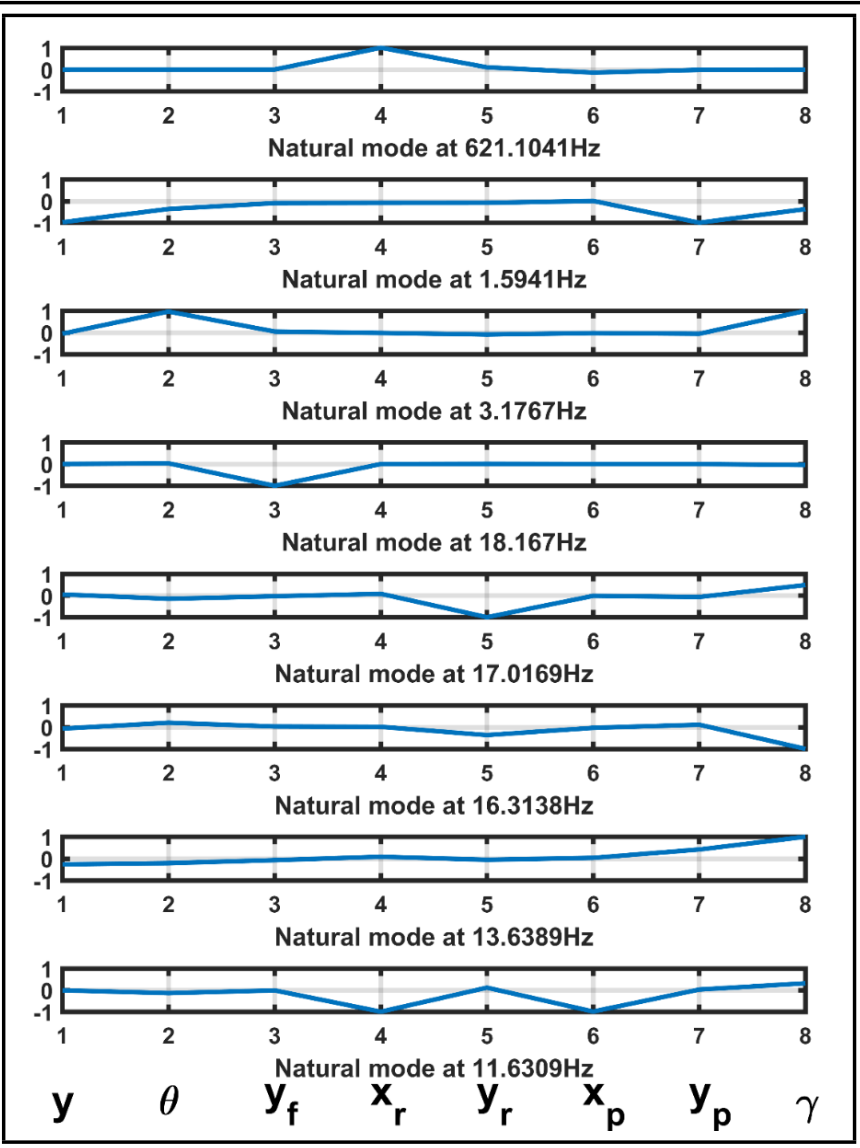

Figure 2. Natural modes_eight degree-of-freedom model.

mounting $(1.65 \mathrm{~Hz}, 16.85 \mathrm{~Hz}, 18.15 \mathrm{~Hz})$ change to $1.59 \mathrm{~Hz}$, $17.01 \mathrm{~Hz}, 18.16 \mathrm{~Hz}$ respectively. However, two of these three modes exhibit a strong pitch component of the powertrain and the first mode also exhibits coupling with sprung mass pitch. Although the frequency for the hop mode of the rear unsprung mass changes only slightly, this mode gets coupled to the powertrain pitch. This is expected to affect the response of the rear tire to any road surface irregularities. Furthermore, this model yields additional frequencies: $3.17 \mathrm{~Hz}, 11.63 \mathrm{~Hz}, 13.64 \mathrm{~Hz}$, $16.32 \mathrm{~Hz}$ and $621.1 \mathrm{~Hz}$. The mode shapes of all the natural modes from this model are shown in Fig. 2.

The mode shapes have been scaled to a maximum magnitude of \pm 1 , and the numbers in the $x$-axis correspond to the eight DOF as listed in Fig. 2. For example, the natural mode at $11.6309 \mathrm{~Hz}$ corresponds to the fore-aft mode of the rear unsprung mass $\left(x_{r}\right)$ coupled to the fore-aft mode of the powertrain $\left(x_{p}\right)$ along with some coupling with the pitch mode of the powertrain $(\gamma)$. This can be seen from the amplitudes of -1 corresponding to $x=4\left(x_{r}\right)$ and $x=6\left(x_{p}\right)$ and an amplitude of 0.2 corresponding to $x=8(\gamma)$ for this mode in Fig. 2. The natural modes imply that for excitation input due to irregularity of the road surface, the lowest natural mode will be excited by a road irregularity of $6 \mathrm{~m}$ wavelength if the motorcycle is traveling at $9.54 \mathrm{~m} / \mathrm{s}$ (or $34.34 \mathrm{kmph})$. However, the highest mode will only be realistically excited by irregularities of very short wavelengths. Incorporating the damping constants yields the damped frequency as well as the damping ratios for each mode. These results are summarized in Table 1.

\subsection{Response to road irregularity}

The model developed in Section 2 is used to analyse the response to different forms of excitation arising from irregu- 


Table 1. Summary of modal analysis results.
\begin{tabular}{|c|c|c|l||}
\hline $\begin{array}{c}\text { Undamped } \\
\text { frequency }(\mathrm{Hz})\end{array}$ & $\begin{array}{c}\text { Damped } \\
\text { frequency }(\mathrm{Hz})\end{array}$ & $\begin{array}{c}\text { Damping } \\
\text { ratio }\end{array}$ & Mode shape \\
\hline 1.59 & 1.57 & 0.158 & Sprung mass bounce coupled with sprung mass pitch, powertrain bounce and powertrain pitch \\
3.17 & 3.04 & 0.283 & Sprung mass pitch coupled with powertrain pitch \\
11.63 & 11.59 & 0.083 & Powertrain fore-aft coupled with rear unsprung fore-aft \\
13.64 & 13.57 & 0.101 & Powertrain pitch with some powertrain bounce \\
16.32 & 16.17 & 0.135 & Powertrain pitch with some rear unsprung hop \\
17.01 & 13.80 & 0.584 & Rear unsprung hop with some powertrain pitch \\
18.16 & 15.25 & 0.543 & Front unsprung hop mode \\
621.10 & 621.09 & 0.005 & Rear unsprung fore-aft mode \\
\hline
\end{tabular}

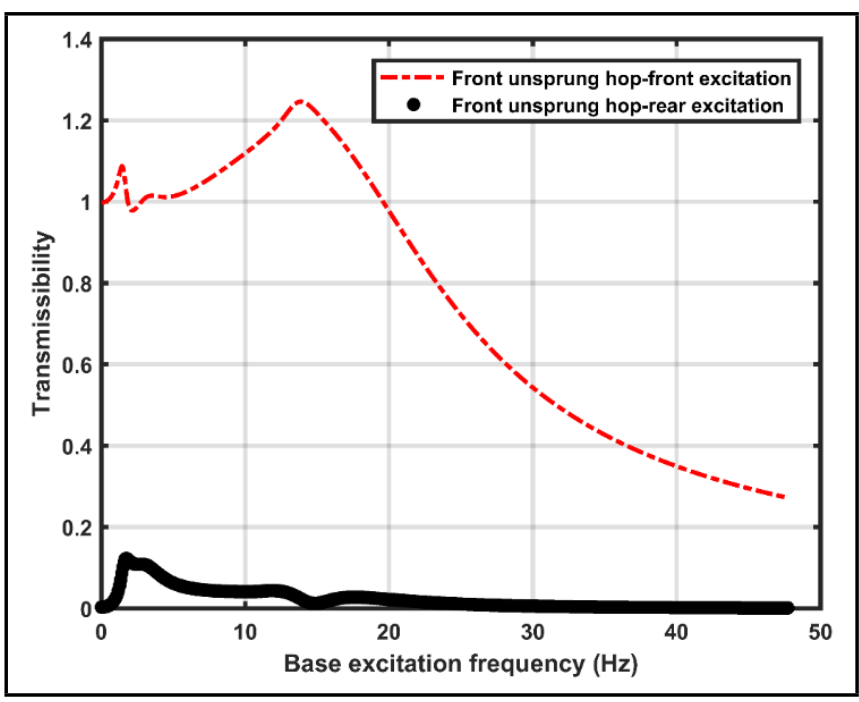

Figure 3. Displacement transmissibility—front unsprung mass (hop).

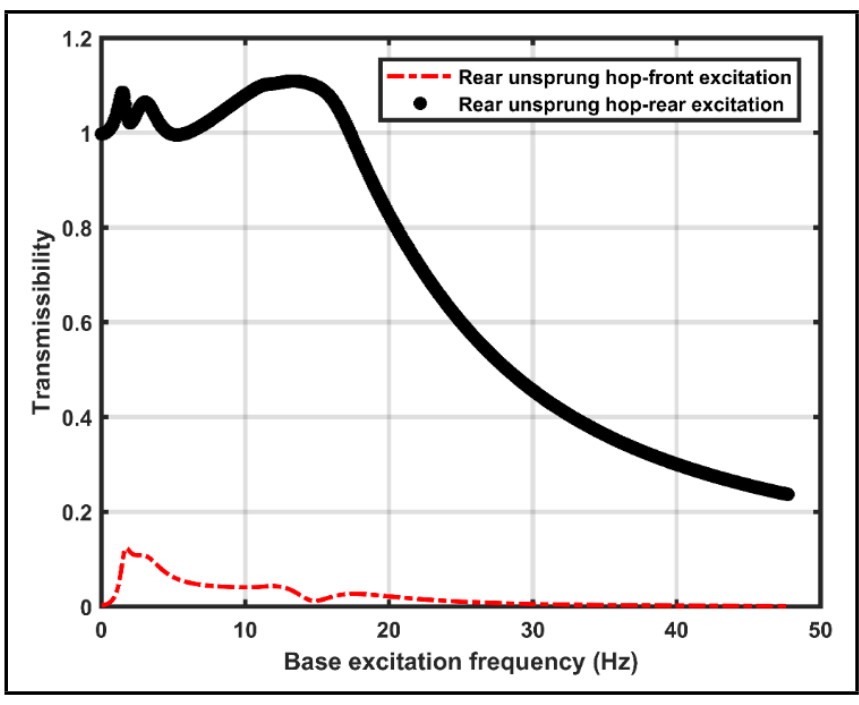

Figure 4. Displacement transmissibility—rear unsprung mass (hop).

larities of the road surface. Road irregularities are modelled simplistically as sinusoidal inputs at the front and rear tire as base excitation, assuming a constant velocity of the motorcycle while traveling in a straight line. A phase difference is introduced in the sinusoidal input between the front and the rear tire to account for the wheelbase separating the two points of contact. A base excitation model is also used to analyse the transmissibility due to excitation resulting from road irregularity.

Figures 3, 4 and 5 show the transmissibility of the front and rear unsprung masses due to excitation at the front and rear tire. The contact points at the front and rear tires have been excited, one at a time, in the form of base excitation with a sinusoidal input with a varying frequency.

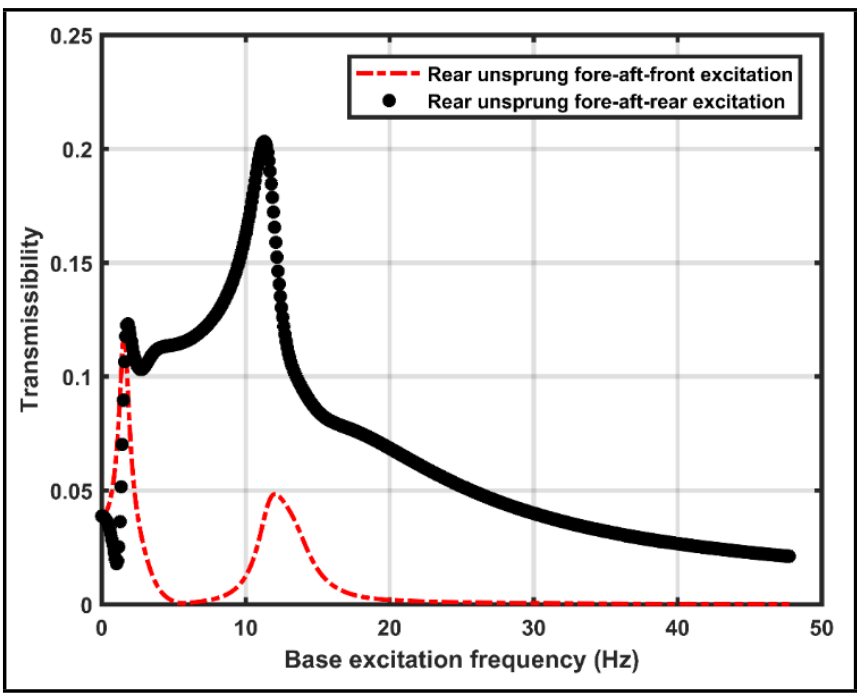

Figure 5. Displacement transmissibility—rear unsprung mass (fore-aft).

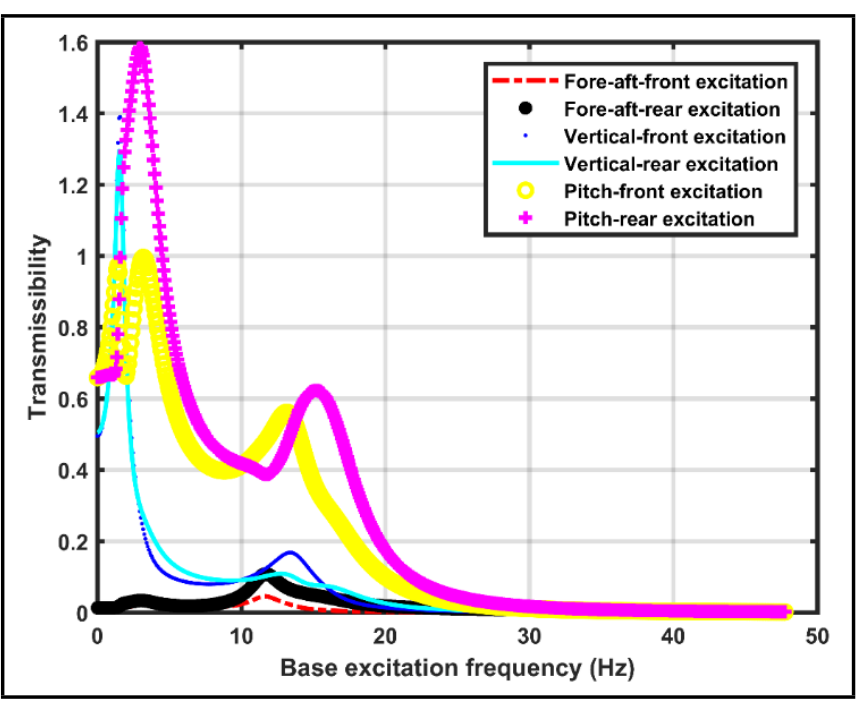

Figure 6. Displacement transmissibility—powertrain.

As can be seen from Fig. 3, the front unsprung mass shows very limited response to any excitation at the rear tire. This phenomenon can also be observed from the response of the rear unsprung mass due to excitation at the front tire, as seen in Fig. 4. However, Fig. 5 exhibits results that are primarily induced by the presence of the isolation system, the rear unsprung mass can be seen to exhibit reasonable fore-aft transmissibility. This could be attributed to the coupled motion between the rear unsprung mass and the powertrain. Figure 6 shows the transmissibility results for the powertrain, it is seen that the transmissibility for the pitch and bounce (vertical motion) is significantly higher due to excitation at the rear tire. This result corresponds with the results of the rear unsprung mass in Fig. 5. Finally, the transmissibility results for the 


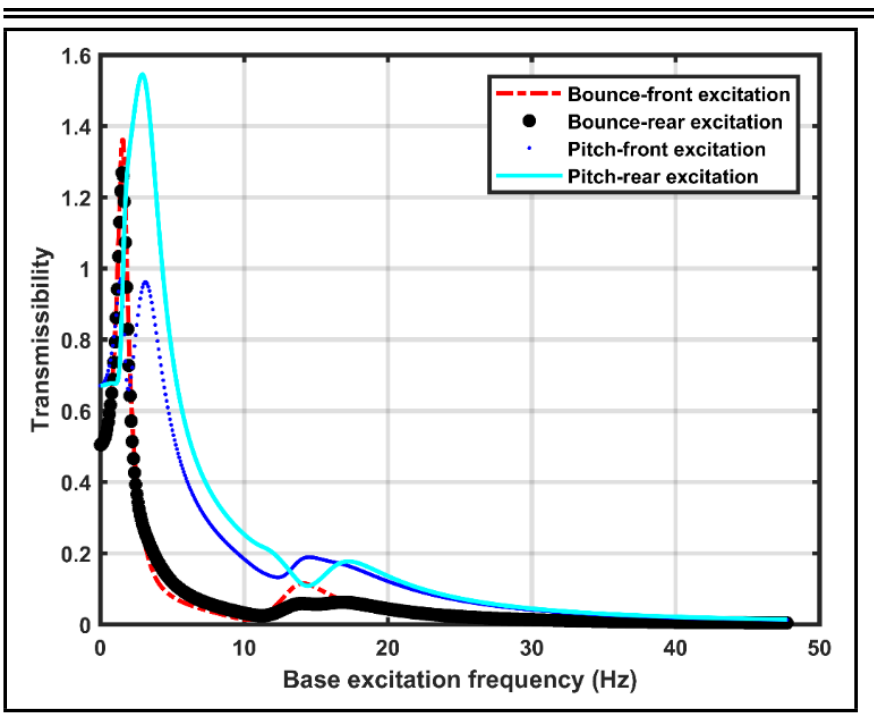

Figure 7. Displacement transmissibility—sprung mass.

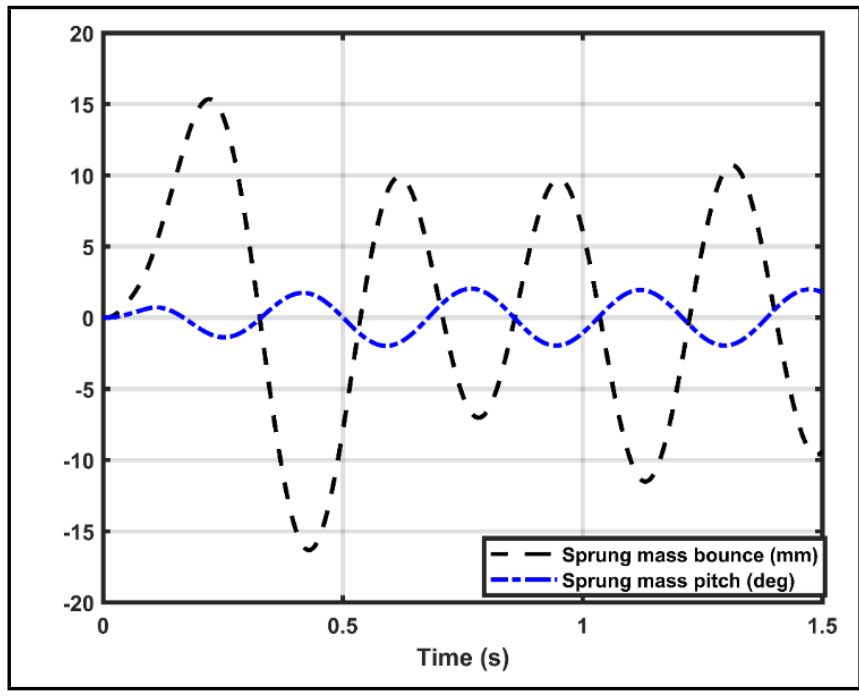

Figure 8. Response—road irregularity—sprung mass.

sprung mass are shown in Fig. 7, these results demonstrate that even though the pitch and bounce transmissibility due to rear excitation is significantly high, transmissibility due to front excitation is not negligible. The comparison between the transmissibility results of the sprung mass and the powertrain indicates a high influence of the vibration isolation system in enhancing ride comfort.

A sinusoidal profile with an amplitude of $20 \mathrm{~mm}$ and a wavelength of $6 \mathrm{~m}$ has been used to model road irregularity, with the motorcycle traveling in a straight line at a constant speed of $17 \mathrm{~m} / \mathrm{s}$ (or $61.2 \mathrm{kmph}$ ). This amounts to a time period of $0.3529 \mathrm{~s}$ for the road profile. The traveling speed has been chosen from the range of $15 \mathrm{~m} / \mathrm{s}$ to $20 \mathrm{~m} / \mathrm{s}$ used in the literature $^{12}$ and can be changed without any loss of generality. It is assumed that both the tires adhere to the road surface, and a time delay is introduced to account for the wheelbase. Also, a point contact is assumed between each tire and the road surface. Displacement results for all the rigid bodies are shown in Figs. 8, 9 and 10, these results are obtained by time integration. The sprung mass bounce is seen to stay below the input amplitude of $20 \mathrm{~mm}$ during the transient response while the pitch is a maximum of 2 deg., as seen in Fig. 8. The response of the two unsprung masses and the powertrain in Figs. 9 and 10 further confirms the coupled motion between the powertrain and

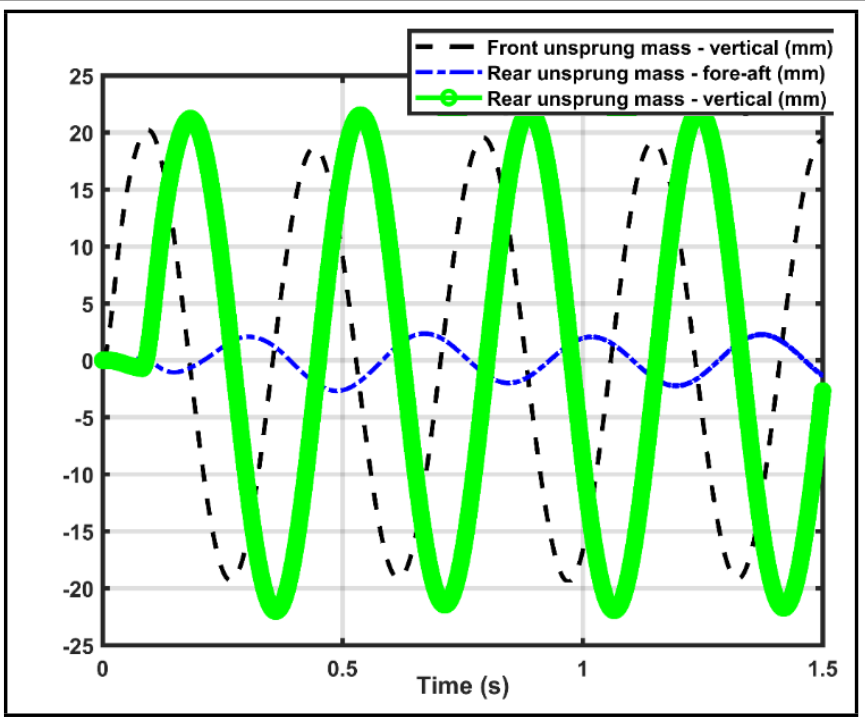

Figure 9. Response—road irregularity—unsprung mass.

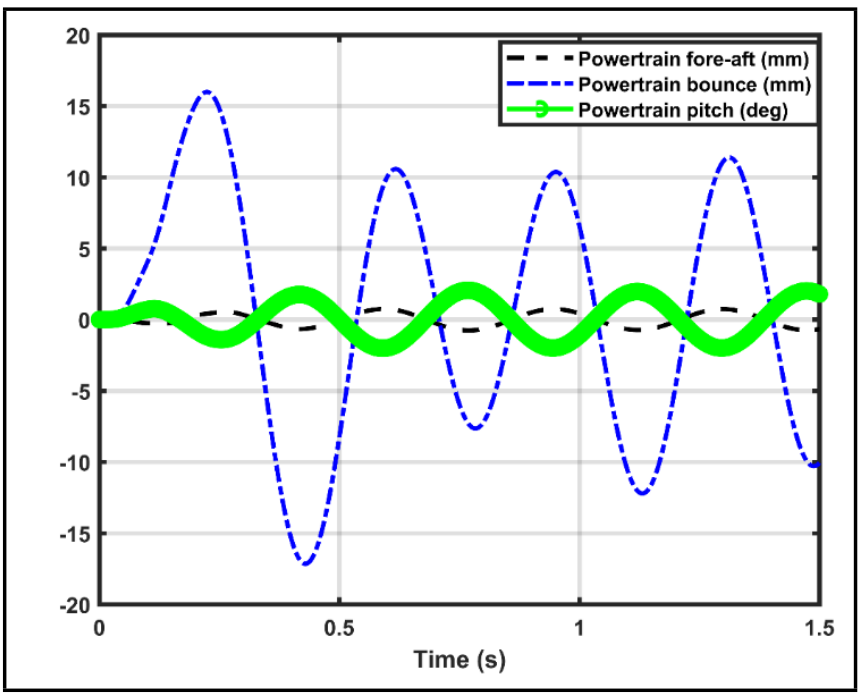

Figure 10. Response-road irregularity-powertrain.

the rear unsprung mass already seen from the transmissibility results. The results from time response further corroborate the enhancement of ride comfort and the potentially negative impact on handling.

Results from the analysis of acceleration PSD are shown in Figs. 11 and 12. A constant speed of $20 \mathrm{~m} / \mathrm{s}$ (or $72 \mathrm{kmph}$ ) is used for this analysis. As expected, the PSD of the horizontal (fore-aft) acceleration is negligible across the frequency spectrum, as seen in Fig. 12. However, the PSD for the vertical (bounce) acceleration of the sprung mass as well as the powertrain is seen to be significant, as seen in Fig. 11 for two different speeds and Fig. 12.

It is important to note that the PSD for pitch accelerations is much higher for the sprung mass as well as the powertrain, however the magnitudes cannot be directly compared due to different units. Also, the PSD for pitch accelerations is seen to have a higher bandwidth whereas the PSD for vertical accelerations drops off sharply. This indicates that the pitch motion of the sprung mass is significantly influenced by the isolation system. The analysis for the sprung mass has been performed at two speeds, $17 \mathrm{~m} / \mathrm{s}$ and $20 \mathrm{~m} / \mathrm{s}$, and the results demonstrate similar trends for sprung mass pitch and bounce, as seen in Figs. 11a and 11b. The PSD for unsprung mass accelerations can be seen in Fig. 13, the front unsprung hop is seen to peak 


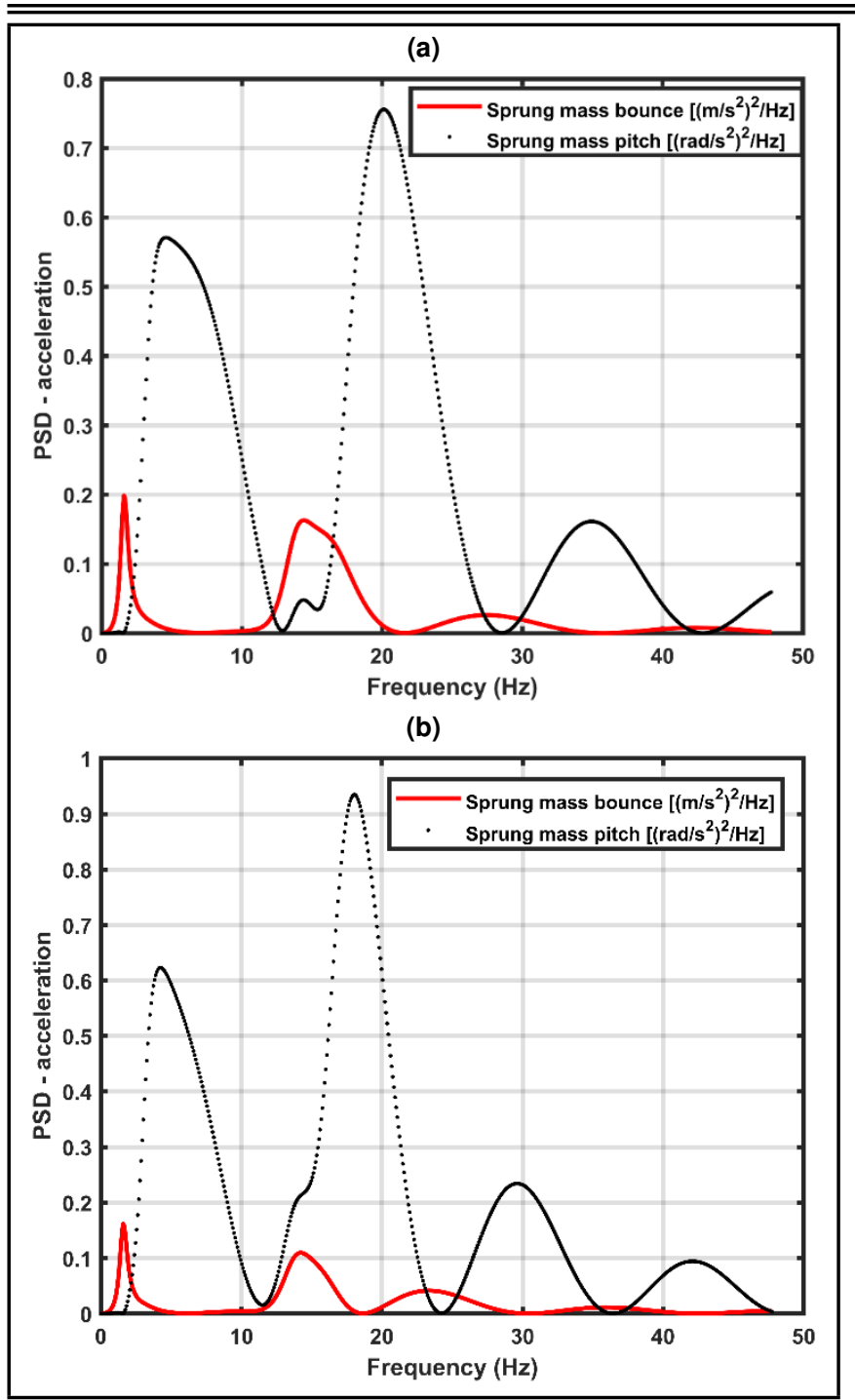

Figure 11. PSD—sprung mass acceleration at constant speed ((a) $20 \mathrm{~m} / \mathrm{s}$, (b) $17 \mathrm{~m} / \mathrm{s}$ ) with isolation system.

at approximately $18 \mathrm{~Hz}$ with a very high bandwidth. The PSD for rear unsprung hop peaks at $16 \mathrm{~Hz}$ with a high bandwidth, as seen in Fig. 13. Figure 14 shows the PSD of accelerations for the sprung mass without a vibration isolation system.

The results in Fig. 14 can be directly compared to Fig. 11a and it can be observed that the use of the vibration isolation system directly results in a substantial increase in the maximum amplitude of the PSD for pitch acceleration. While the PSD for bounce acceleration of the sprung mass does not change significantly, the bandwidth is higher when the isolation system is used. This result could have significant implications on the assessment of ride comfort. The PSD for unsprung mass accelerations without an isolation system is shown in Fig. 15, these results can be directly compared to the results in Fig. 13.

It is observed that the isolation system does not have much influence on the amplitude or bandwidth of the front unsprung hop acceleration but the peak shifts from 18 to $22 \mathrm{~Hz}$. On the other hand, the rear unsprung hop acceleration exhibits a shift in its peak (from 15 to $20 \mathrm{~Hz}$ ) and a change in bandwidth, as can be seen by comparing the results of Fig. 13 and Fig. 15. This can have implications for the handling behaviour of the motorcycle and will need to be assessed through model validation and testing.

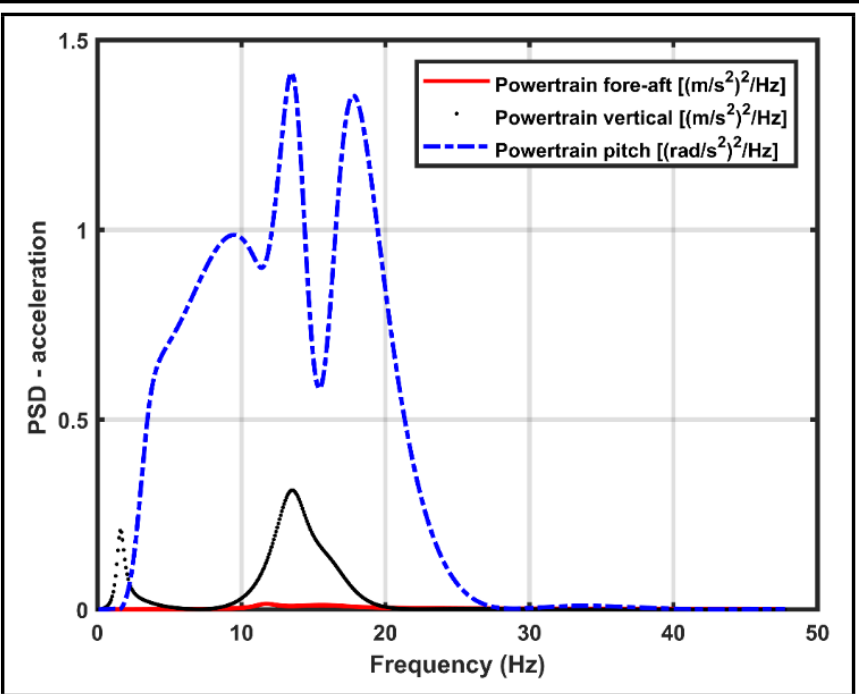

Figure 12. PSD_-powertrain acceleration at constant speed $(20 \mathrm{~m} / \mathrm{s})$ with isolation system.

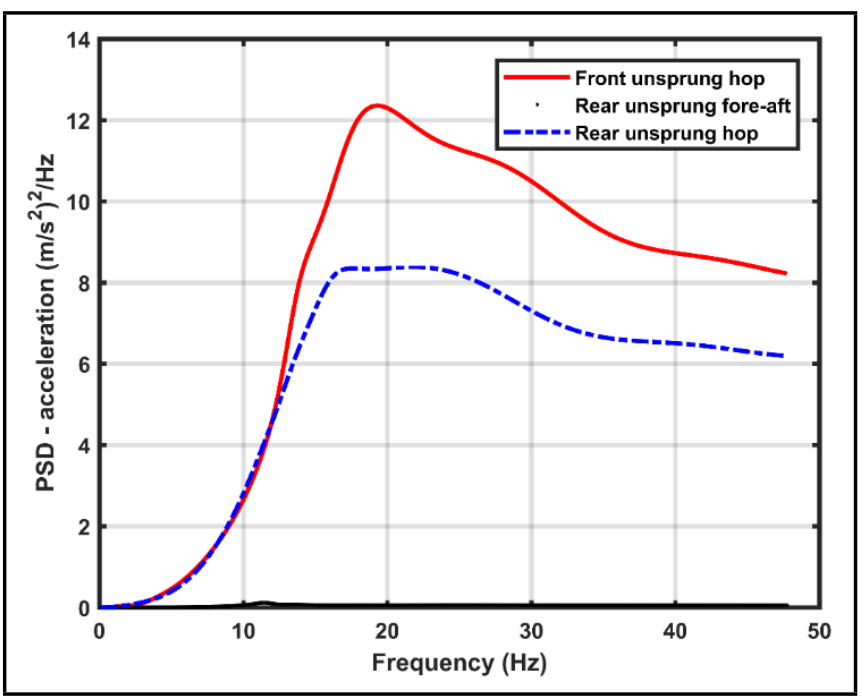

Figure 13. PSD - unsprung mass accelerations at constant speed $(20 \mathrm{~m} / \mathrm{s})$ with isolation system.

\section{CONCLUSIONS}

In this paper, the influence of a vibration isolation system on the in-plane dynamics of a motorcycle has been evaluated. An eight DOF model has been developed for analysis, and the model is used to determine the system's response to road irregularities and to compute the natural modes. Although the layout of the isolation system can vary from one design to another, the model presented in this paper represents one possible arrangement that can be modified to accommodate other designs. While transmissibility results indicate that the isolation system enhances ride comfort, the isolation system couples the rear unsprung hop with the pitch of the powertrain. The natural frequency associated with the rear unsprung hop is seen to increase by $1 \%$ while exhibiting a coupling with the powertrain pitch, this would increase the response to road irregularities of relatively shorter wavelengths (or higher frequencies) as compared to a rigidly mounted powertrain. Furthermore, the modes associated with the powertrain pitch are lightly damped. This is expected to result in relatively higher amplitudes for the powertrain pitch. These results indicate that while the isolation system enhances ride comfort, it may adversely affect in-plane handling. 


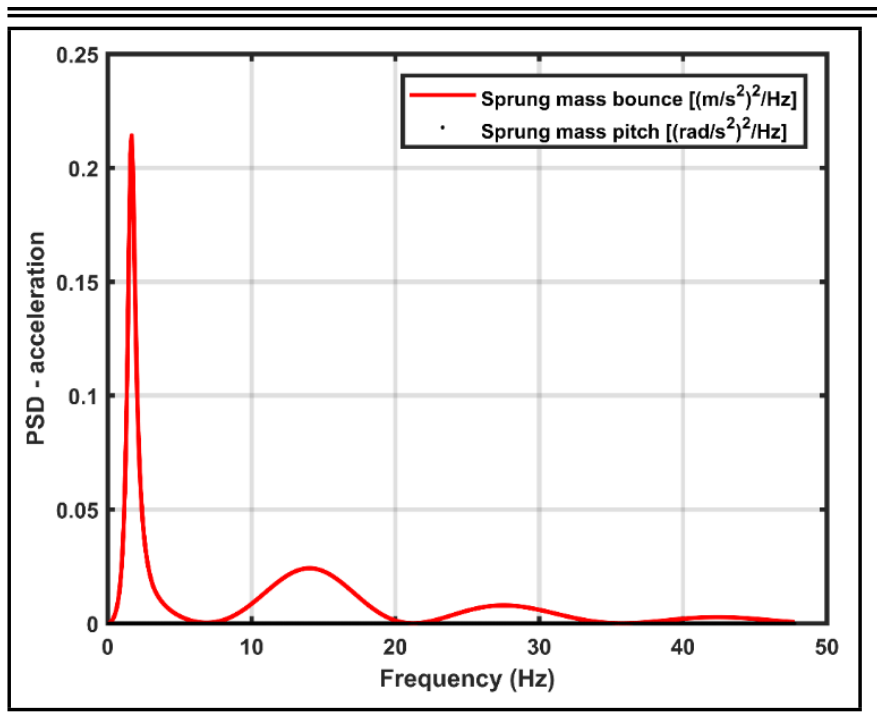

Figure 14. PSD—sprung mass acceleration at constant speed $(20 \mathrm{~m} / \mathrm{s})$ without isolation system.

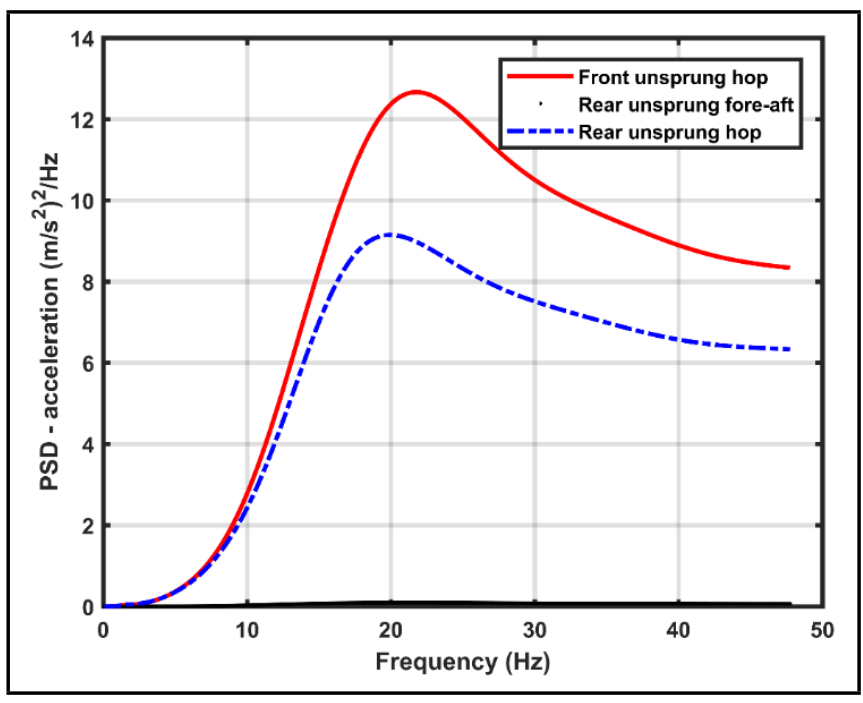

Figure 15. PSD—unsprung mass accelerations at constant speed $(20 \mathrm{~m} / \mathrm{s})$ without isolation system.

The power spectral density results demonstrate that the isolation system causes a significant increase in the PSD of pitch acceleration of the sprung mass. However, the PSD of bounce acceleration of the sprung mass exhibits a limited influence due to the isolation system. In comparison to a rigidly mounted system, the use of an isolation system is seen to affect the peak and bandwidth of the PSD of hop acceleration of the rear unsprung mass, thereby directly affecting the handling behaviour. Although the analysis in this study has been limited to rectilinear motion at steady speed, these results indicate that the introduction of the vibration isolation system may adversely influence the handling of the motorcycle. This needs to be taken into careful consideration during the design of the isolation system.

The results obtained in this study are based on a relatively simple model and, therefore, need to be experimentally and analytically validated. The model developed in this study can be used to comprehend the sensitivity of in-plane dynamics to specific parameters. Future work will focus on investigating the influence of different isolation systems and suspension systems on in-plane dynamics. The model will be used for design optimization in order to balance the functional needs of ride comfort and handling.

\section{REFERENCES}

1 Sharp, R. S., Evangelou, S., and Limebeer, D. J. N. Advances in the modelling of motorcycle dynamics, Multibody System Dynamics, 12, 251-283, (2004). https://dx.doi.org/10.1023/B:MUBO.0000049195.60868.a2

2 Sharp, R. S. Optimal linear time-invariant preview steering control for motorcycles, Vehicle System Dynamics, 44, 329-340, (2006). https://dx.doi.org/10.1080/00423110600871509

3 Cossalter, V., Doria, A., Garbin, S., and Lot, R. Frequencydomain method for evaluating the ride comfort of a motorcycle, Vehicle System Dynamics, 44, 339-355, (2006). https://dx.doi.org/10.1080/00423110500420712

4 Cossalter, V. and Lot, R. A Motorcycle multi-body model for real time simulations based on the natural coordinates approach, Vehicle System Dynamics, 37, 423-447, (2002). https://dx.doi.org/10.1076/vesd.37.6.423.3523

5 Limebeer, D. and Sharp, R. S. Bicycles, motorcycles and models, IEEE Control Systems Magazine, 26, 34-61, (2006). https://dx.doi.org/10.1109/mcs.2006.1700044

6 Mechanical Simulation Corp, BikeSim, Ann Arbor, MI, USA, (2017). Retrieved from carsim.come.

7 Dynamotion Srl, FastBike, Padova, Italy, (2017).

8 Ricardo, L. A. R. Nonlinear Stochastic Analysis of Motorcycle Dynamics, $\mathrm{PhD}$ Thesis, Houston, TX, USA, Rice University, (2013). Retrieved from lhdl.handle.net/1911/72032.

9 Kaul, S. and Dhingra A. K. Engine mount optimization for vibration isolation in motorcycles, Vehicle System Dynamics, 47, 419-436, (2009). https://dx.doi.org/10.1080/00423110802167458

10 Kaul, S., Dhingra, A. K., and Hunter, T. G. Frame flexibility effects on engine mount optimization for vibration isolation in motorcycles, Journal of Vibration and Acoustics, 129, 590-600, (2007). https://dx.doi.org/10.1115/1.2748468

11 Cocco, G. Motorcycle Design and Technology, Giorgio Nada, Vimodrone, Italy, (2001).

12 Cossalter, V. Motorcycle Dynamics, Second Edition, Lulu, Morrisville, NC, USA, (2006).

13 Kaul, S. Multi-degree-of-freedom modeling of mechanical snubbing systems, Journal of Vibroengineering, 13, 195211, (2011). https://dx.doi.org/10.1115/DETC2011-47144

14 Roe, G. E. and Thorpe, T. E. The influence of frame structure on the dynamics of motorcycle stability, SAE Small Engine Technology: Conference Proceedings, Paper 891772, (1989). https://dx.doi.org/10.4271/891772

15 Sun, L. On human perception and evaluation to road surfaces, Journal of Sound and Vibration, 247, 547-560, (2001). https://dx.doi.org/10.1006/jsvi.2001.3642 